\title{
Nonlinear Registration of Diffusion MR Images Based on Fiber Bundles ${ }^{\star}$
}

\author{
Ulas Ziyan ${ }^{1}$, Mert R. Sabuncu ${ }^{1}$, Lauren J. O'Donnell ${ }^{2,3}$, \\ and Carl-Fredrik Westin ${ }^{1,3}$ \\ ${ }^{1}$ MIT Computer Science and Artificial Intelligence Lab, Cambridge MA, USA \\ ulas@mit .edu \\ ${ }^{2}$ Department of Neurosurgery, Brigham and Women's Hospital, Harvard Medical \\ School, Boston MA, USA \\ ${ }^{3}$ Laboratory of Mathematics in Imaging, Brigham and Women's Hospital, \\ Harvard Medical School, Boston MA, USA
}

\begin{abstract}
In this paper, we explore the use of fiber bundles extracted from diffusion MR images for a nonlinear registration algorithm. We employ a white matter atlas to automatically label major fiber bundles and to establish correspondence between subjects. We propose a polyaffine framework to calculate a smooth and invertible nonlinear warp field based on these correspondences, and derive an analytical solution for the reorientation of the tensor fields under the polyaffine transformation. We demonstrate our algorithm on a group of subjects and show that it performs comparable to a higher dimensional nonrigid registration algorithm.
\end{abstract}

\section{Introduction}

Diffusion tensor imaging (DTI) measures the molecular diffusion, i.e., Brownian motion, of the endogenous water in tissue. This water diffusion is anisotropic in fibrous biological tissues such as cerebral white matter. Quantification of water diffusion in tissue through DTI provides a unique way to look into white matter organization of the brain [1].

Tractography is a common post-processing technique for DTI which aims at reconstructing fibers from the tensor field [2]. The method works by tracing the principal diffusion direction in small steps. The resulting tracts can be grouped together into bundles. Even though the resolution of DTI is too low to measure any individual axons, these bundles show similarity to anatomical structures and suggest that at the bundle level we are able to capture structural information [3, 4.

A popular technique to investigate white matter anatomy is to manually select regions of interest (ROIs) that are thought to correspond to a particular anatomical white matter tract, and to analyze scalar measures derived from the diffusion

\footnotetext{
* This work was supported by NIH NIBIB NAMIC U54-EB005149, NIH NCRR NAC P41-RR13218, R01-MH074794 and the Athinoula A. Martinos Foundation. We are grateful to Susumu Mori at JHU for the diffusion MRI data (R01-AG20012 / P41RR15241) and Serdar Balci for the ITK help.
} 
tensors within the ROI. ROI based methods could be subject to user bias if the ROIs are manually traced. Therefore several methods have been proposed to identify anatomically meaningful regions from the DTI data. One class of methods uses tractography results and groups them into regions either interactively or automatically, e.g. 3, 4. A recent study reported that tractography-based definitions of a pyramidal tract ROI are more reproducible than manual ROI drawing [5].

An alternative to ROI-based studies is to perform spatial normalization for the whole data set followed by voxel based morphometry in the white matter. There are many registration techniques designed for aligning scalar MR images such as structural T1 weighted images. Some of these linear and non-linear methods have been applied to scalar images derived from the diffusion tensor data 6, 7 , 8, 9, 10.

However, DTI is by nature a non-scalar image and potentially offers rich information that can be used to determine voxel-wise correspondence across subjects. This has been observed in some studies and resulted in: 1) multi-channel scalar registration techniques that aim to account for orientational information, and 2) correspondence validation techniques that are not not only based on voxel-wise similarities but also on fiber tracts generated from the DTI data sets.

The contributions of this paper are multi-fold. Firstly, we propose a novel algorithm to non-linearly register DTI data sets (of multiple subjects) based on fiber bundles that have been automatically segmented. We employ a polyaffine framework [1] to fuse the bundle-based transformations. This yields a global, one-to-one nonlinear deformation. Secondly, we derive an analytical solution for the reorientation of the tensor fields after the application of the polyaffine deformation. Finally, we propose a new measure (of fiber-tensor fit, FiT) to quantify the quality of the match between a deformed fiber tract and underlying diffusion tensor field.

\section{Methods}

\subsection{Fiber Bundles}

Organization of tract fibers into bundles, in the entire white matter, reveals anatomical connections such as the corpus callosum and corona radiata. By simultaneously clustering fibers of multiple subjects into bundles, major white matter structures can be discovered in an automatic way 12. The results of such a clustering can then saved as bundle models along with expert anatomical labels to form a white matter atlas, which can later be used to label tractography results of new subjects 12 .

In this work, we use such an atlas to automatically label tractography results of a group of subjects. Since the labeling is consistent among multiple subjects, the use of such an atlas not only provides individual's label maps but also correspondence between subjects. As described in the following Section, we employ this correspondence determined by the label maps to perform nonlinear inter-subject registration. 


\subsection{Registration of Fiber Bundles}

Inter-subject registration using fiber tracts is not a well studied problem. To our knowledge, the only study that deals with this question performs registration on a tract by tract basis utilizing a rigid invariant tract parametrization [13. The drawback of this framework is that it only allows for rigid transformations (with 6 independent variables) and is computationally very expensive due to the need to process fibers individually.

Instead of registering tracts individually, we propose to register corresponding bundles. To align the bundles from two subjects, we compute 3 dimensional spatial probability maps for each fiber tract bundle in each subject. The goal of our registration is to maximize the correlation between two corresponding bundles' probability maps. A fiber bundle $b_{i}$ consists of a set of tracts $\left\{t_{j}\right\}$, where $t_{j}$ is represented by a set of points $\left\{\boldsymbol{x}_{k}\right\}$. Given a fiber bundle we define $b_{i}$ 's spatial probability map as:

$$
P_{b_{i}}(\boldsymbol{x})=\frac{1}{Z} \sum_{t_{j} \in b_{i}} \sum_{\boldsymbol{x}_{k} \in t_{j}} \kappa\left(\boldsymbol{x}-\boldsymbol{x}_{k}\right),
$$

where $Z$ is the appropriate normalization for a valid probability density (calculated by summing the estimated spatial probability map), and $\kappa\left(\boldsymbol{x}-\boldsymbol{x}_{k}\right)$ is a (Gaussian) kernel centered around $\boldsymbol{x}_{k}$, the $k$ th sample from the tract $t_{j}$.

Next, we discretize these probability maps to obtain a 3D scalar image for each tract bundle in each subject. We employ a sequential quadratic programming method [14] to find the 9 affine parameters that maximize the correlation coefficient between corresponding probability maps in different subjects. For a pair of subjects, this yields a set of affine transformations that relate each corresponding bundle pair. The last step of our algorithm is to fuse these affine transformations to achieve a global, invertible nonlinear deformation. There are several ways to achieve this. In this study, we employed the log-Eucledian polyaffine framework [11] which guarantees invertibility.

\subsection{Polyaffine Framework}

The log-Euclidean polyaffine framework offers a fast method to calculate an invertible and smooth nonlinear warp field with a small number of parameters [11. matrices that define three dimensional affine transformations in homogeneous coordinates. Abusing notation, we denote the affine transformation defined by the matrix $\boldsymbol{A}$ as a vector valued function $A(\boldsymbol{x}) \in \mathbb{R}^{3}$ for $\boldsymbol{x} \in \mathbb{R}^{3}$. Our goal is to obtain a global deformation field, $\Phi(\boldsymbol{x})$, that is computed by a weighted fusion of these affine transformations $\left(A_{i}\right.$ 's). The most obvious way to combine these transformations is through a weighted sum, but that does not in general yield a smooth and invertible deformation [11. One way of achieving a well behaved deformation is to use the following stationary ordinary differential equation:

$$
\frac{d}{d t} \boldsymbol{x}(t)=\sum_{i=1}^{S} w_{i}(\boldsymbol{x}(t)) \log \left(\boldsymbol{A}_{i}\right)(\boldsymbol{x}(t))
$$


with $\boldsymbol{x}(0)=\boldsymbol{x}_{0}$ and $\log \left(\boldsymbol{A}_{i}\right) \in \mathbb{R}^{4 \times 4}$ is the principal logarithm of $\boldsymbol{A}_{i}$. Note $\log \left(\boldsymbol{A}_{i}\right)(\boldsymbol{x})$ is an affine transformation. The fused transformation is defined as: $\Phi\left(\boldsymbol{x}_{0}\right)=\boldsymbol{x}(1)$. A numerical solution to (1) is computed in the following manner. Define:

$$
T_{N}(\boldsymbol{x})=\sum_{i=1}^{S} w_{i}(\boldsymbol{x}) A_{i}^{2^{-N}}(\boldsymbol{x}),
$$

where $N$ is a small positive integer (typically 4 or 5 ), $\boldsymbol{A}_{i}^{2^{-N}}$ denotes the $N$ th square root of $\boldsymbol{A}_{i}$ and the weights sum up to one, i.e., $\sum_{i} w_{i}(\boldsymbol{x})=1$ for all $\boldsymbol{x}$. Note that $A_{i}^{2^{-N}}(\boldsymbol{x})$ is also an affine transformation. The global transformation $\Phi$ is then obtained in $\mathrm{N}$ steps:

$$
\Phi(\boldsymbol{x})=\underbrace{\left[T_{1} \circ T_{2} \circ \cdots \circ T_{N}\right]}_{N \text { times }} \circ T_{N}(\boldsymbol{x}),
$$

where $T_{n}=T_{n+1} \circ T_{n+1}$, for $n \in\{1, \ldots, N-1\}$, and $\circ$ denotes concatenation. The inverse of $\Phi(\boldsymbol{x})$ is also calculated using the same formulation by replacing each $A_{i}$ with $A_{i}^{-1}$ in Equation 2 11.

\subsection{Diffusion Tensor Rotation}

Deforming a tensor field is not as easy as interpolating the tensors at their new locations. Since they carry directional information, diffusion tensors need to be rotated when they undergo a spatial transformation [6]. Given the rotational component $\boldsymbol{R}$ of the transformation, the tensors $\boldsymbol{D}$ should be reoriented to $\boldsymbol{R}^{T} \boldsymbol{D} \boldsymbol{R}$. This rotational component is readily available in an affine transformation. However, it needs to be estimated for a nonlinear deformation field. One way to do so is to calculate the deformation gradient tensor (i.e., Jacobian matrix) $\boldsymbol{J}_{\Phi}(\boldsymbol{x}) \in \mathbb{R}^{3 \times 3}$ of the deformation $\Phi$. This captures the locally linear component of the deformation and can be employed via the finite strain method [6] to estimate the rotational component: $\boldsymbol{R}=\left(\boldsymbol{J} \boldsymbol{J}^{T}\right)^{-1 / 2} \boldsymbol{J}$. For an arbitrary deformation field the Jacobian can be approximated with finite differences. We used this method for the nonlinear benchmark algorithm in Section 3.

An interesting property of the polyaffine framework is that an analytic expression for the Jacobian can be derived. Let's re-write $A_{i}^{2^{-N}}$ from (2) as $A_{i}^{2^{-N}}(\boldsymbol{x})=$ $\boldsymbol{M}_{\boldsymbol{i}} \boldsymbol{x}+\boldsymbol{t}_{i}$. Notice that $T_{N}(\boldsymbol{x})=\sum_{i=1}^{S} w_{i}(\boldsymbol{x}) \boldsymbol{M}_{\boldsymbol{i}} \boldsymbol{x}+w_{i}(\boldsymbol{x}) \boldsymbol{t}_{i}$. Then the Jacobian of $T_{N}$ is:

$$
\begin{aligned}
\boldsymbol{J}_{T_{N}}(\boldsymbol{x}) & =\sum_{i=1}^{S} w_{i}(\boldsymbol{x}) \boldsymbol{M}_{i}+\left(\boldsymbol{M}_{i} \boldsymbol{x}+\boldsymbol{t}\right)\left(\nabla w_{i}(\boldsymbol{x})\right)^{T} \\
& =\sum_{i=1}^{S} w_{i}(\boldsymbol{x}) \boldsymbol{M}_{i}+T(\boldsymbol{x})\left(\nabla w_{i}(\boldsymbol{x})\right)^{T}
\end{aligned}
$$

where.$^{T}$ denotes transpose. Given $\boldsymbol{J}_{T_{N}}(\boldsymbol{x})$ and using (3), the Jacobian of $\Phi$ is computed with the chain rule in $N$ steps: 


$$
\boldsymbol{J}_{\phi(\boldsymbol{x})}=\underbrace{\left[\boldsymbol{J}_{T_{1}}\left(T_{2}(\boldsymbol{x})\right) \cdots \boldsymbol{J}_{T_{N-1}}\left(T_{N}(\boldsymbol{x})\right) \cdot \boldsymbol{J}_{T_{N}}\left(T_{N}(\boldsymbol{x})\right)\right]}_{N \text { times }} \cdot \boldsymbol{J}_{T_{N}}(\boldsymbol{x}),
$$

where $\boldsymbol{J}_{T_{n}}=\boldsymbol{J}_{T_{n+1}}\left(T_{n+1}(\boldsymbol{x})\right) \cdot \boldsymbol{J}_{T_{n+1}}(\boldsymbol{x})$, for $n \in\{1, \ldots, N-1\}, \boldsymbol{J}_{T_{N}}($.$) is defined$ in (4) and $\cdot$ is matrix multiplication.

\subsection{Measure for Registration Quality: FiT}

Two diffusion tensor images can be considered well registered if the tracts generated from one data set match well with the other data set's tensor field after deformation. To quantify this, we propose a measure we name Fiber-Tensor Fit (FiT).

Given a three dimensional probability density $p(\boldsymbol{x})$, its orientation distribution function $(\mathrm{ODF})$ is defined as [15]:

$$
\psi(\boldsymbol{u})=\int_{0}^{\infty} p(\boldsymbol{u} r) d r,
$$

where $\boldsymbol{u} \in \mathbb{R}^{3}$ is a unit vector and $r$ is a scalar parameter (the radius in polar coordinates).

In diffusion imaging, water diffusion is commonly modeled with a Gaussian distribution. $\boldsymbol{D}(\boldsymbol{x})$ be the diffusion tensor at that location. The ODF at $\boldsymbol{x}$ can be written as:

$$
\begin{aligned}
\psi(\boldsymbol{u}(\boldsymbol{x})) & =\int_{0}^{\infty} \frac{1}{\sqrt{(2 \pi)^{3}|\boldsymbol{D}(\boldsymbol{x})|}} \exp \left(-\frac{1}{2} r \boldsymbol{u}(\boldsymbol{x})^{T} \boldsymbol{D}^{-1}(\boldsymbol{x}) \boldsymbol{u}(\boldsymbol{x}) r\right) d r \\
& =\frac{1}{\sqrt{(4 \pi)^{2}|\boldsymbol{D}(\boldsymbol{x})|}} \frac{1}{\sqrt{\boldsymbol{u}(\boldsymbol{x})^{T} \boldsymbol{D}^{-1}(\boldsymbol{x}) \boldsymbol{u}(\boldsymbol{x})}}
\end{aligned}
$$

Note that for a given a diffusion tensor $\boldsymbol{D}(\boldsymbol{x})$, the ODF is upper bounded: $\psi(\boldsymbol{u}(\boldsymbol{x})) \leq \sqrt{\lambda_{1}(\boldsymbol{x})} / \sqrt{(4 \pi)^{2}|\boldsymbol{D}(\boldsymbol{x})|}$, where $\lambda_{1}(\boldsymbol{x})$ denotes the maximum eigenvalue of $\boldsymbol{D}(\boldsymbol{x})$. The tract ODF value at $\boldsymbol{x}$ is defined as $\psi\left(\boldsymbol{t}_{i}(\boldsymbol{x})\right)$, where $\boldsymbol{t}_{i}(\boldsymbol{x})$ denote the tangent vector to a fiber tract $t_{i}$ at $\boldsymbol{x}$. We define FiT as the sum of the $\log$ ratios of the tract ODF values to the maximum ODF values. Thus, ignoring constants, the FiT of a fiber tract $t_{i}$ on a tensor field $\boldsymbol{D}$ is defined as:

$$
\varphi\left(t_{i} ; \boldsymbol{D}\right)=-\left(\sum_{\boldsymbol{x} \in t_{i}} \log \left(\boldsymbol{t}_{i}(\boldsymbol{x})^{T} \boldsymbol{D}^{-1}(\boldsymbol{x}) \boldsymbol{t}_{i}(\boldsymbol{x})\right)+\log \left(\lambda_{1}(\boldsymbol{x})\right)\right) .
$$

Notice that $\varphi\left(t_{i} ; \boldsymbol{D}\right)$ is not sensitive to an arbitrary ordering of eigenvectors that have very close eigenvalues. Also, the value of FiT is maximized for the first-order streamline tractography solution, since that would mean a perfect alignment between the tract tangent vector and the principle eigenvector at every sample along the tract. Even though FiT is well suited for quantifying the fiber-tensor alignment, a direct optimization of this measure may not be feasible since the algorithmic complexity of each iteration would be the same as running a full brain tractography. 


\section{Experiments}

We analyzed 15 full brain Diffusion Tensor MR images of resolution $2.5 \times 2.5 \times$ $2.5 \mathrm{~mm}$. Tractography was performed in each subject using Runge-Kutta order two integration. As a benchmark, we employed an ITK implementation [16] of an FA-based non-linear registration method (known as the demons algorithm) that has been successfully applied to DTI data 7. This method performs a dense matching on the intensity images using a diffusion process. Regularization is achieved through Gaussian smoothing.

Selecting the first subject as the template, we provide results for 14 pairwise registrations using: 1) an FA-based global affine registration algorithm [17, 2) the demons algorithm, and 3) the proposed bundle-based polyaffine algorithm. Figure 1includes 3D renderings of the registered tracts from a subject (in green)
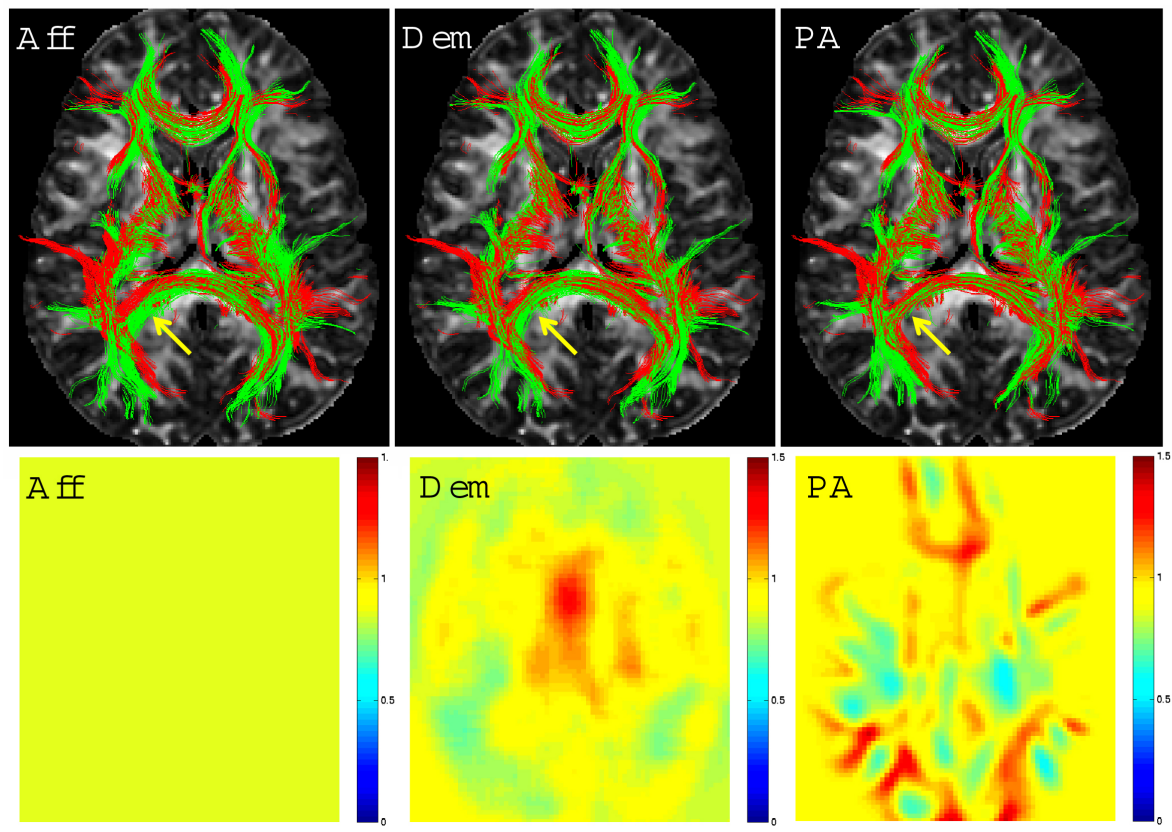

Fig. 1. Top Row: 3D renderings of the registered tracts of a subject (in green) and the template (in red) within $\pm 5 \mathrm{~mm}$ of the central axial slice overlayed on the central FA slice of the template. "Aff" (left) stands for the FA based global affine, "Dem" (middle) for the demons algorithm and "PA" (right) for the polyaffine framework as proposed in this work. Arrows point to an area of differing qualities of registration. Overlapping of the red and green fibers is indicative of better registration. Bottom Row: Jacobian determinant images from the central slice of the volume: Yellow represents areas with small changes in size, and the shades of red and blue represent enlargement and shrinking, respectively. The Jacobian of the global affine registration is constant. The Jacobian of the demons algorithm is smooth due to the Gaussian regularization. The Jacobian of the polyaffine algorithm reflects the underlying anatomy because of the fiber bundle-based definition of the deformation. 


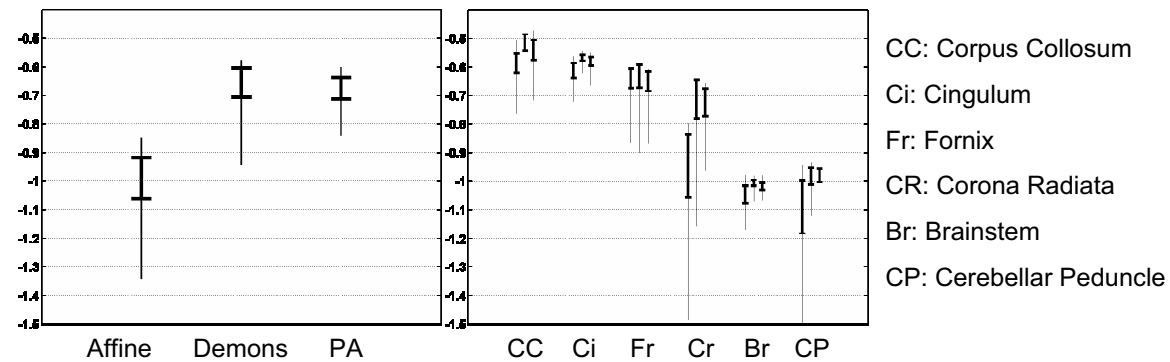

Fig. 2. Left: Mean FiT values from 14 subjects' DTI sets. Each subject's DTI data is aligned with the template and compared with the template's fiber tracts. Thin lines indicate the range of the FiT values, and the thick crossbars indicate the regions within one standard deviation from the mean. Right: Mean FiT values from 14 subjects' major fiber bundles. For each bundle, the left error bar is for affine; the middle one is for demons and the right one is polyaffine. Relative ranges of the fit values for major structures are similar to the averages from the whole data sets, however differ from each other in absolute terms.

and the template tracts (in red) overlayed on the central axial FA slice of the template. The second row shows the central slice of the Jacobian determinant volumes for the corresponding deformations computed by each registration algorithm. Note that certain anatomical structures are visible in the proposed algorithm's Jacobian image. This is due to the bundle-based definition of the underlying deformation field. The Jacobian image of the demons algorithm, however, demonstrates no clear relationship with the underlying anatomy. A close investigation of the fiber tract renderings in Figure 1 reveals that both non-linear algorithms, in general, achieve significantly better alignment of the tracts. There are some regions in this image, e.g. the corpus callosum, where the bundle-based algorithm yields more accurate matching than the demons algorithm. Figure 2 includes plots of average FiT values for the different algorithms and some major bundles of interest. The nonlinear algorithms achieve consistently better registrations than to the affine algorithm. The two nonlinear algorithms yield comparable results.

\section{Discussion}

This paper explores an inter-subject bundle-based nonlinear registration algorithm for DTI data sets. The algorithm performs comparable to a higher dimensional nonrigid registration algorithm, and it has certain advantages that many nonlinear algorithms lack, such as the ease of calculating the inverse transform. We also showed that there is an analytic expression for the Jacobian matrix of our deformation field, which was used for the reorientation of the deformed diffusion tensor field. Furthermore, the quality of the registration can be improved 
with higher degrees of freedom, such as multiple affine components per structure, without losing the cited advantages.

\section{References}

[1] Basser, P.J., Mattiello, J., Bihan, D.L.: MR diffusion tensor spectroscopy and imaging. Biophys. J. 66, 259-267 (1994)

[2] Basser, P., Pajevic, S., Pierpaoli, C., Duda, J., Aldroubi, A.: In vivo fiber tractography using DT-MRI data. Magnetic Resonance in Medicine 44, 625-632 (2000)

[3] O'Donnell, L., Westin, C.F.: White matter tract clustering and correspondence in populations. In: Duncan, J.S., Gerig, G. (eds.) MICCAI 2005. LNCS, vol. 3749, pp. 140-147. Springer, Heidelberg (2005)

[4] Maddah, M., Mewes, A., Haker, S., Grimson, W.E.L., Warfield, S.: Automated atlasbased clustering of white matter fiber tracts from DTMRI. In: Duncan, J.S., Gerig, G. (eds.) MICCAI 2005. LNCS, vol. 3749, pp. 188-195. Springer, Heidelberg (2005)

[5] Partridge, S.C., Mukherjee, P., Berman, J.I., Henry, R.G., Miller, S.P., Lu, Y., Glenn, O.A., Ferriero, D.M., Barkovich, A.J., Vigneron, D.B.: Tractography-based quantitation of diffusion tensor imaging parameters in white matter tracts of preterm newborns. Magnetic Resonance in Medicine 22(4), 467-474 (2005)

[6] Alexander, D.C., Pierpaoli, C., Basser, P.J., Gee, J.C.: Spatial transformations of diffusion tensor magnetic resonance images. IEEE TMI 20 (2001)

[7] Park, H.J., Kubicki, M., Shenton, M.E., Guimond, A., McCarley, R.W., Maier, S.E., Kikinis, R., Jolesz, F.A., Westin, C.-F.: Spatial normalization of diffusion tensor MRI using multiple channels. Neuroimage 20(4), 1995-2009 (2003)

[8] Jones, D.K., Griffin, L., Alexander, D., Catani, M., Horsfield, M., Howard, R., Williams, S.: Spatial normalization and averaging of diffusion tensor MRI data sets. Neuroimage 17(2), 592-617 (2002)

[9] Leemans, A., Sijbers, J., Backer, S.D., Vandervliet, E., Parizel, P.M.: Affine coregistration of diffusion tensor magnetic resonance images using mutual information. In: Blanc-Talon, J., Philips, W., Popescu, D.C., Scheunders, P. (eds.) ACIVS 2005. LNCS, vol. 3708, pp. 523-530. Springer, Heidelberg (2005)

[10] Xu, D., Mori, S., Shen, D., van Zijl, P.C., Davatzikos, C.: Spatial normalization of diffusion tensor fields. Magnetic Resonance in Medicine 50(1), 175-182 (2003)

[11] Arsigny, V., Commowick, O., Pennec, X., Ayache, N.: A fast and Log-Euclidean polyaffine framework for locally affine registration. Research report RR-5865, INRIA Sophia-Antipolis (2006)

[12] O'Donnell, L., Westin, C.F.: High-dimensional white matter atlas generation and group analysis. In: Larsen, R., Nielsen, M., Sporring, J. (eds.) MICCAI 2006. LNCS, vol. 4191, pp. 243-251. Springer, Heidelberg (2006)

[13] Leemans, A., Sijbers, J., Backer, S.D., Vandervliet, E., Parizel, P.M.: Multiscale white matter fiber tract coregistration: a new feature-based approach to align diffusion tensor data. Magnetic Resonance in Medicine 55(6), 1414-1423 (2006)

[14] Fletcher, R., Powell, M.: A rapidly convergent descent method for minimization. Computer Journal 6, 163-168 (1963)

[15] Tuch, D.S., Reese, T.G., Wiegell, M.R., Wedeen, V.J.: Diffusion MRI of complex neural architecture. Neuron 40, 885-895 (2003)

[16] Ibanez, L., Schroeder, W., Ng, L., Cates, J.: The ITK Software Guide. 1st edn. Kitware, Inc. ISBN 1-930934-10-6. (2003)

[17] Zollei, L., Learned-Miller, E., Grimson, W.E.L., Wells III, W.M.: Efficient population registration of $3 \mathrm{D}$ data. In: ICCV, Computer Vision for Biomedical Image Applications (2005) 